\title{
Le tuyau bonna : un siècle de présence à l'étranger
}

\author{
Bernard André
}

Société des Tuyaux Bonna

\section{I — LA SOCIÉTÉ DES TUYAUX BONNA}

La Société des Tuyaux Bonna doit son nom à un ingénieur de génie, Aimé Bonna, qui invente et fabrique dès juillet 1894 le premier tuyau à tube médian en acier avec double revêtement en béton armé, système qui porte encore le nom de son inventeur. Dès 1900, Aimé Bonna se tourne vers les marchés étrangers : Belgique, Angleterre, Espagne où il réalise une canalisation spectaculaire pour l'époque: $2000 \mathrm{ml}$ de diamètre $3850 \mathrm{~mm}$.
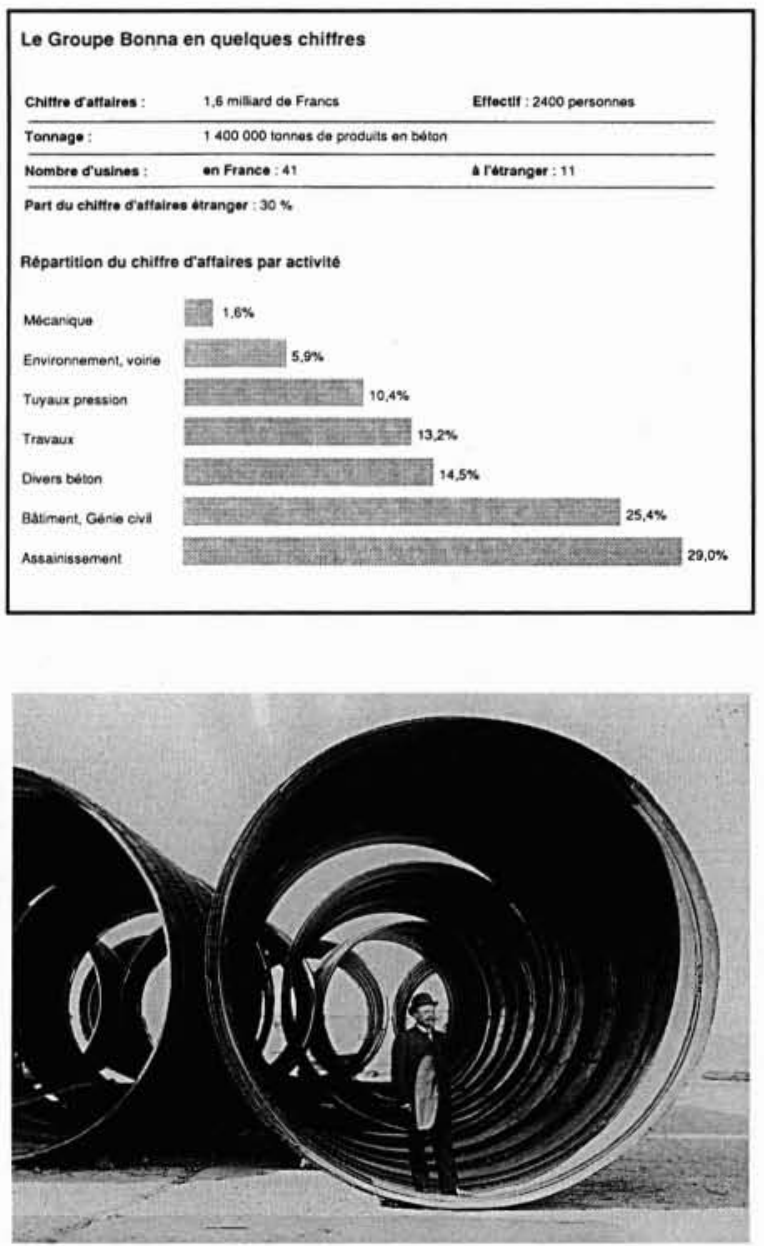

1. Siphon du Río Sosa (Espagne) 1905.
Le 24 novembre 1924, les Etablissements Aimé Bonna deviennent la Société des Tuyaux Bonna, filiale de la Compagnie Générale des Eaux.

Depuis la date de sa création, la Société des Tuyaux Bonna a fabriqué plus de $25000 \mathrm{~km}$ de tuyaux sous pression (tuyaux à tube médian en acier et double revêtement en béton armé ou tuyaux en béton précontraint) dont une large partie, en dehors du territoire français, a été posée par elle-même dans plus de trente pays différents. De la conception à la réalisation clés en main, Bonna module son action en fonction des services qui lui sont demandés.

\subsection{Une vocation multiple}

Bonna apporte son expérience, son assistance technique et sa technologie réunies au sein d'une organisation industrielle intégrée qui comprend:

- des départements spécialisés dans l'étude et la conception d'unités de production de tuyaux, dans l'ingénierie et la conception des réseaux de canalisations ;

- des usines de fabrication : 52 dont 11 à l'étranger ;

- des départements Travaux chargés du Génie Civil et de la pose de canalisations. A l'étranger, la tâche de ces équipes peut être totale ou partielle allant de l'envoi de superviseurs à la réalisation complète de la pose ;

- un département commercial situé à Paris La Défense ;

- des départements commerciaux régionaux.

\section{II — LES TUYAUX BONNA}

2.1 Le tuyau pression en béton armé avec âme en tôle

C'est le tuyau Bonna classique connu dans le monde entier depuis 1894. Il existe dans des diamètres allant de 300 à $4000 \mathrm{~mm}$ et comprend :

- un tube médian en tôle d'acier mince terminé par des bagues d'about conformées suivant le type de joint prévu ;

- un revêtement intérieur en béton assurant la protection interne du tube médian et capable de supporter des débits très élevés :

- un revêtement extérieur en béton armé.

Que ce soit dans le domaine de l'adduction d'eau sous pression ou celui des amenées d'eau dans le secteur industriel, la fiabilité et la pérennité du réseau sont primordiales et supposent une véritable ingénierie du projet. 
En conjugant intimement les performances de l'acier et du béton, en tirant parti le plus élaboré de l'association de ces deux matériaux. Bonna, avec le tuyau à âme en tôle, fournit à ses clients depuis des décennies les moyens de leurs projets d’alimentation en eau. La constante amélioration des procédés de production, l'accumulation continue d'un développement sur les cinq continents permettent à notre société d'offrir un produit dont les qualités intrinsèques en font le champion de longévité et d'adaptibilité aux besoins de ses clients.

Il est désormais fabriqué en conformité avec la nouvelle norme européenne Pr EN 641 et sous la Certification ISO 9002 .

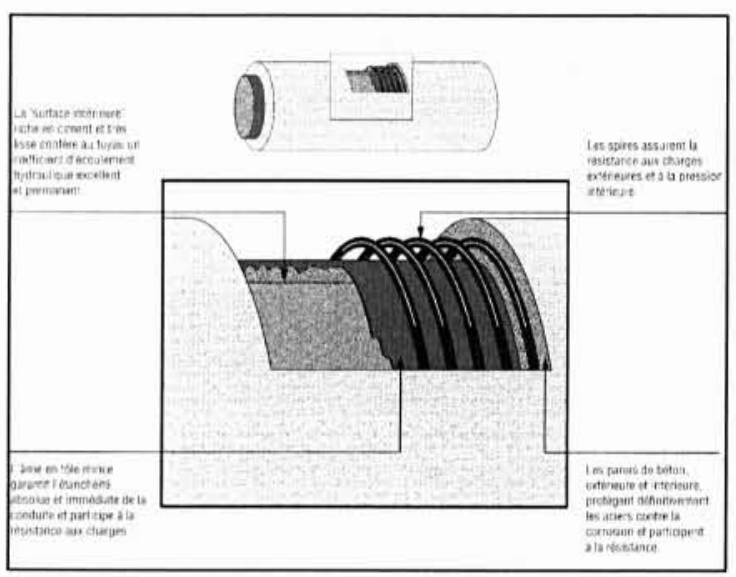

2. Le tuyau à âme en tôle.

\subsection{Le tuyau à écoulement libre en béton armé}

Bonna fabrique également des tuyaux centrifugés compactés ROCLA ainsi que des tuyaux moulés vibrés BVA pour l'assainissement.

Face au développement de la technique des microtunnels, un tuyau qui répond aux exigences de fonçage par microtunnelier a été mis au point. La mise en cuvre du tuyau par centrifugation et son durcissement en moule ont permis d'obtenir une qualité d'aspect nettement supérieure et surtout d'obtenir un Béton Haute Performance (700 à 900 bars). L'étanchéité est obtenue par joint intégré dans l'about femelle.

L'expérience acquise avec les tuyaux pour microtunnels et une analyse en profondeur menée auprès des Maîtres d'CEuvre et d'Ouvrages ont abouti au développement d'un nouveau tuyau pour pose en tranchée, le BOHP. Deux innovations majeures au niveau de la performance du béton et de la conception de l'emboîtement assurent aux réseaux d'assainissement une étanchéité pérenne. Ces tuyaux sont garantis 10 ans.

\subsection{Le tuyau en béton précontraint}

D'autres types de tuyaux pression mieux adaptés à certains types de projets ou plus économiques ont été mis au point selon les conditions locales:

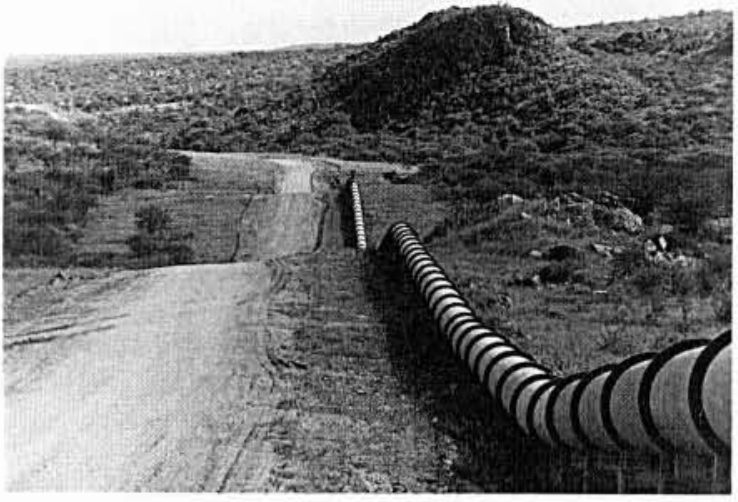

3. Conduite de Omatako-Von Bach (Namibie).

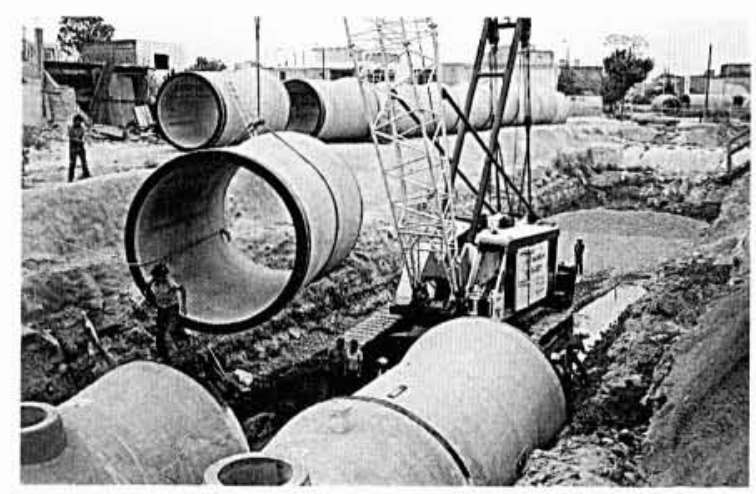

4. Alimentation en eau de Guadalajara (Mexique). Tuyaux en béton précontraint.

- tuyaux en béton précontraint dits frettés sur tôle incorporée possédant généralement, comme le tuyau âme tôle classique, un joint soudé :

- tuyaux en béton précontraint sans âme en tôle utilisés pour les canalisations portant sur de longues distances et dont les conditions de pose ne présentent pas de difficultés liées à la configuration du terrain.

\section{LES GRANDS CHANTIERS D'ADDUC- TION ET D'IRRIGATION}

Paris et sa banlieue, Lyon, Marseille, Bruxelles, Vancouver, Swansea, Rio de Janeiro, Dakar, Le Caire, Manille, Ankara, Istanbul... la plupart des grandes villes du monde possèdent d'importantes conduites d'adduction d'eau réalisées en tuyaux Bonna. Certaines de ces conduites sont bientôt centenaires. Elles démontrent combien la pérennité du tuyau à âme en tôle est un critère déterminant dans le choix d'une conduite. La possibilité d'utiliser des joints soudés permet d'éviter les massifs de butée au droit des pièces spéciales et de réduire l'emprise de la conduite. L'implantation parmi les autres réseaux s'en trouve facilitée.

Pour le transport d'eau sur de longues distances, les tuyaux en béton précontraint avec ou sans âme ont été également utilisés. 
En Afrique Australe, par exemple, Bonna a participé à la construction d'une conduite en béton précontraint de 120 $\mathrm{km}$ pour la ville de Port Elizabeth, puis, elle a créé avec SOCEA, une société en participation, Southern Pipeline Contractors, qui a fabriqué dans son usine de Kliprivier les conduites d'alimentation en eau pour les villes de Durban, Le Cap, Johannesbourg... SPC a également réalisé l'adduction d'eau de Windhoek (Namibie), l'une des plus longues du monde.

\section{IV — L'EXPORTATION ET L'ÉQUIPEMENT DE SITES INDUSTRIELS}

Les caractéristiques du tuyau à âme en tôle en font un matériau particulièrement bien adapté aux conduites installées dans des sites industriels.

Dès 1927, débute la fabrication de tuyaux de diamètre $2500 \mathrm{~mm}$. Grâce à ce tuyau, Bonna devient l'un des fournisseurs privilégiés de ce qui deviendra EDF.

Le refroidissement du condenseur des centrales électriques nécessite d'importantes quantités d'eau amenées le plus souvent par des canalisations de très grands diamètres, de 1000 à $3000 \mathrm{~mm}$, voire plus.

Ces installations exigent des canalisations d'amenée et de retour d'eau d'une sécurité absolue, ne nécessitant ni entretien, ni réparation.

Bonna a participé à l'équipement des circuits de refroidissement de la plupart des centrales nucléaires en France et d'une cinquantaine de centrales thermiques en France et à l'étranger.

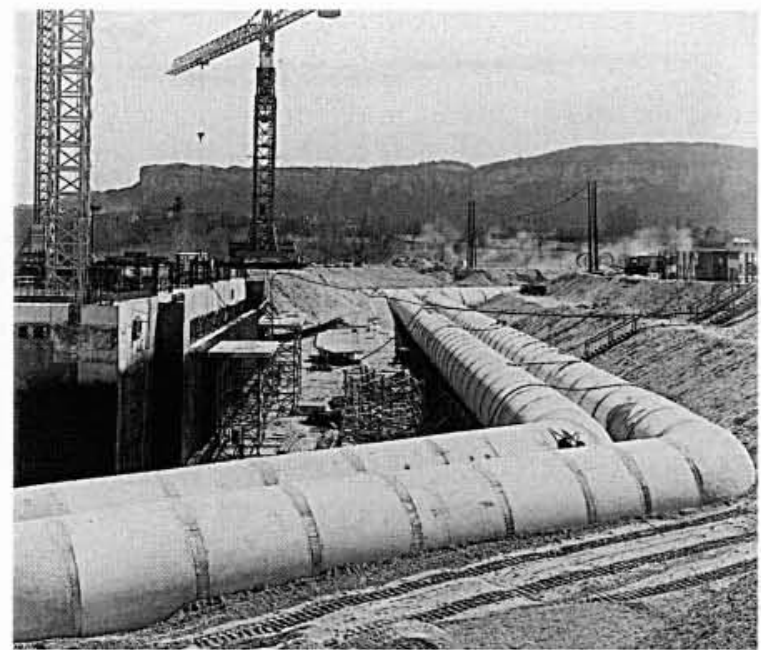

5. Conduite d'amenée et de rejet de la centrale nucléaire EDF-Le Bugey.

Avant 1970, nous participons aux projets clés en main de Bureaux d'Etudes français qui incorporent notre technique dans leurs réalisations à l'étranger: Alsthom en Nouvelle-Calédonie, en Grèce, en Corée du Sud, Heurtey en Algérie, etc. Vers 1970, l'ouverture financière engendrée par le premier choc pétrolier permet aux pays producteurs de pétrole de mettre en auvre des programmes ambitieux dans les domaines de l'énergie, de la pétrochimie, des

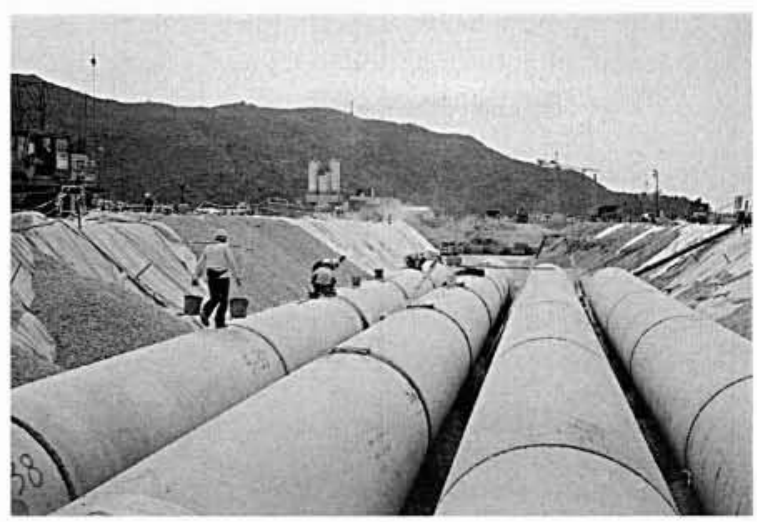

6. Centrale de Black Point (Hong Kong). Rejointoiement extérieur.

engrais et du dessalement d'eau de mer. Au total, plus de $300 \mathrm{~km}$ de conduites à âme en tôle de diamètres 300 à $2800 \mathrm{~mm}$ ont été exportés à ce jour vers le Moyen Orient : Arabie Saoudite, Algérie, Bahrein, Egypte, Emirats Arabes Unis, Irak, Iran, Jordanie, Koweit, Libye, Liban, Maroc, Pakistan, Qatar, Syrie, Tunisie... et aussi vers la Chine, l'Indonésie, Hong Kong, Cuba, La Martinique, le Nigéria...

En 1995, Bonna participera à l'équipement de plusieurs centrales électriques, dont: celle de Paka, en Malaisie, celle de Tambak Lorok, en Indonésie, la centrale thermique de Bin Qasim au Pakistan, la centrale électrique de Rades, en Tunisie, celle de Boxberg en Allemagne et celle de South Humber Bank en Angleterre. Les tuyaux sont soit exportés de France, soit fabriqués localement, selon les cas.

\section{$\mathrm{V}$ ASSAINISSEMENT}

Optimisation des composants, innovation technologique, contrôle qualité, Bonna met au service de l'assainissement un système complet de tuyaux et regards de visite et des produits d'une haute technologie capables de répondre aux contraintes de l'environnement.

A l'étranger, pour suivre l'évolution de leurs marchés, un certain nombre de filiales de production créées à l'origine dans le cadre d'importants projets d'adduction d'eau se sont diversifiées avec des produits d'assainissement. C'est le cas de la société COMECOP au Mexique, STF en Espagne, SPC en Afrique du Sud...

En Egypte, ECPC, spécialisée dans la production de tuyaux en béton précontraint avec âme en tôle pour des projets d'alimentation en eau et d'assainissement sous pression, vient d'obtenir une commande de 72000 voussoirs pour revêtir les parois d'un collecteur d'eaux usées venant se brancher sur le collecteur principal auquel ECPC a participé il y a bientôt 10 ans, pour la ville du Caire.

\section{VI —LES POSES SPÉCIFIQUES}

A partir d'un plan d'implantation sommaire, nos bureaux d'études réalisent un plan d'appareillage complet grâce auquel tuyaux et pièces spéciales à âme en tôle seront parfaitement adaptés au tracé de la conduite. 


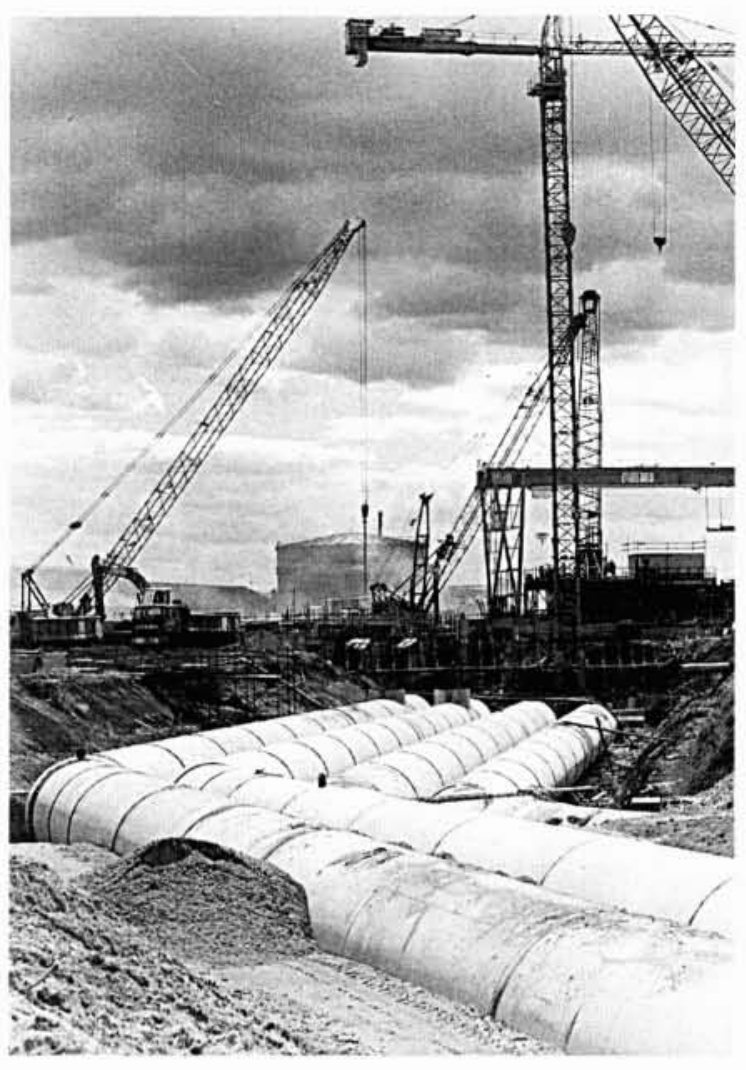

7. Circuit de refroidissement de la centrale de Barking Reach (Grande-Bretagne).

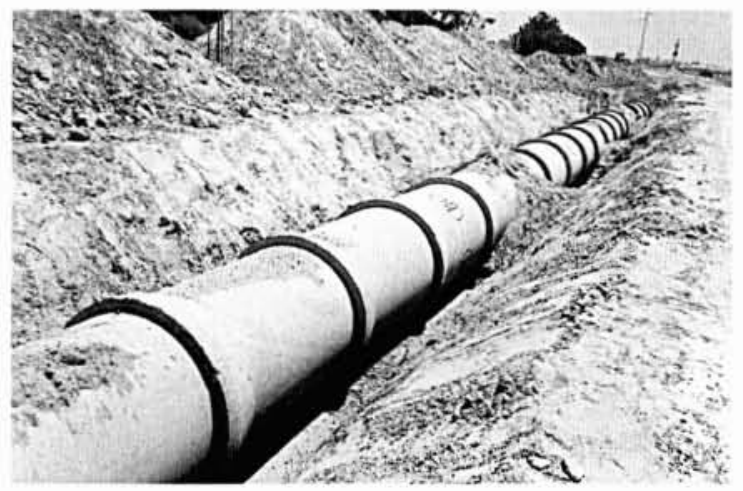

8. Assainissement de Isla Antilla (Espagne).

Si des obstacles sont à franchir, diverses solutions sont envisagées :

- fonçage de la conduite.

- pose en gaine.

- tuyau poutre.

- pose sous-fluviale ou sous-marine..

Bonna a une renommée certaine dans l'exécution de travaux de pose spécifiques.

\subsection{Fonçage}

Pour passer sous des obstacles naturels ou industriels existants, Bonna a développé la technique du fonçage par vérin: un procédé Bonna sans nuisance et de grande sécurité.

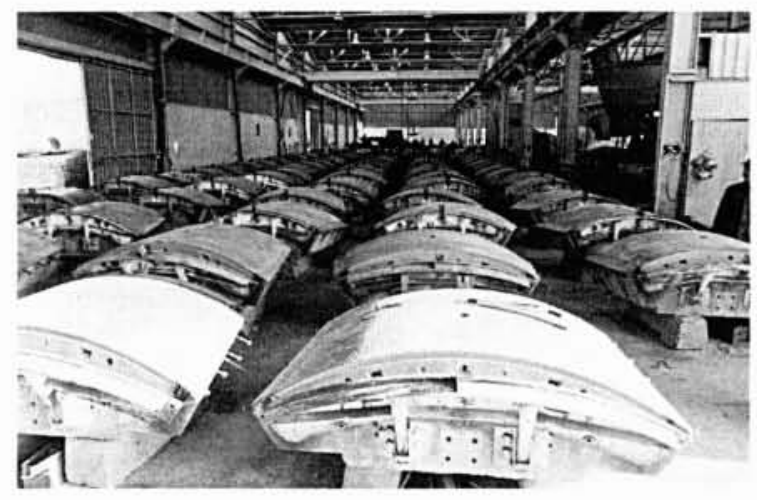

9. Hall de fabrication de voussoirs (Egypte).

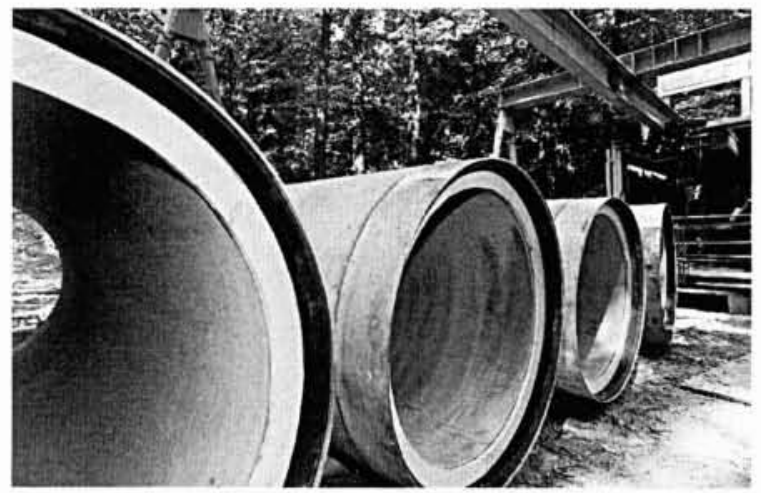

10. Tuyaux pour microfonçage.

\subsection{Pose sous l'eau}

Pour les prises d'eau, les rejets (émissaires) en mer ou les traversées de rivière, les différentes techniques de pose sont adaptées aux contraintes de chaque chantier. Bonna conçoit également les tuyaux à mettre en cuvre (renforcement, poids...) dans chaque cas.

\subsection{Pose en site industriel}

La pérennité du béton, alliée à la souplesse d'adaptation des techniques Bonna à tous les stades, bureau d'études. préfabrication, assistance technique, mise en œuvre et suivi des chantiers lui ont permis de réaliser la plupart des circuits de refroidissement des centrales nucléaires françaises et de nombreux sites industriels en France et à l'étranger.

6.4 Pose en site urbain, une nouvelle technologie, les microtunnels

Ils permettent la suppression des tranchées à ciel ouvert et des nuisances pour l'environnement. Bonna vient de mettre au point des tuyaux spécifiques dont les hautes performances sont étudiées pour résister aux efforts de poussée importants des microtunneliers. 


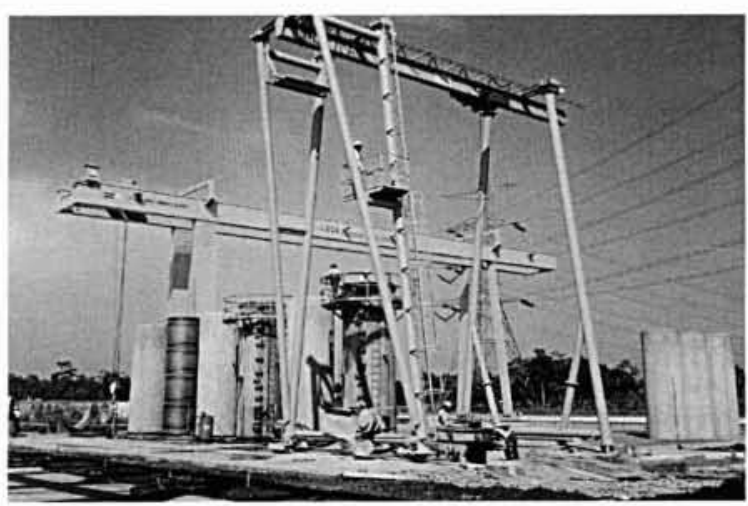

11. Site de production de HBI en Indonésie.

\subsection{Réhabiliter, l'autre solution}

Pour rénover des conduites anciennes, une large gamme de procédés ou techniques est utilisée pour conserver la conduite existante en occasionnant un minimum de gêne aux riverains et à moindre coût.

Parmi les solutions proposées:

- le gainage Paltem,

- les tubages acier, PVC ou par coques GRC et GRP,

- l'injection de résines,

- le procédé Tubolining.

A l'étranger, notre filiale anglaise, Stanton-Bonna, fabrique des coques et des tuyaux GRP utilisés en GrandeBretagne mais aussi pour des projets de réhabilitation en France.

\section{UN SERVICE CLÉS EN MAIN}

A l'étranger, Bonna soumissionne seule ou en association avec des sociétés françaises ou étrangères pour l'exécution de travaux de canalisations de grande envergure qui, dépassant le cadre de l'exportation, l'amènent à créer une ou plusieurs usines de fabrication dans les pays où elle opère.

Une réalisation exemplaire : l'adduction d'eau d'Ankara et d'Istanbul. En 1968, l'Administration turque décide d'augmenter les capacités du réseau d'adduction d'eau des villes d'Ankara et d'Istanbul qui connaissent alors un essor démographique et économique très important. Bonna obtient la fourniture et la pose de $80 \mathrm{~km}$ de conduites en béton précontraint, de diamètre 1850 à 2200 , et s'engage à construire deux usines, à Tuzla et à Aydinkoï, qui devront, à l'issue du marché, rester la propriété du maître d'œuvre. Vingt ans plus tard, grâce à des relations commerciales suivies, Bonna se voit confier un contrat d'ingénierie pour la modernisation de ces deux usines.

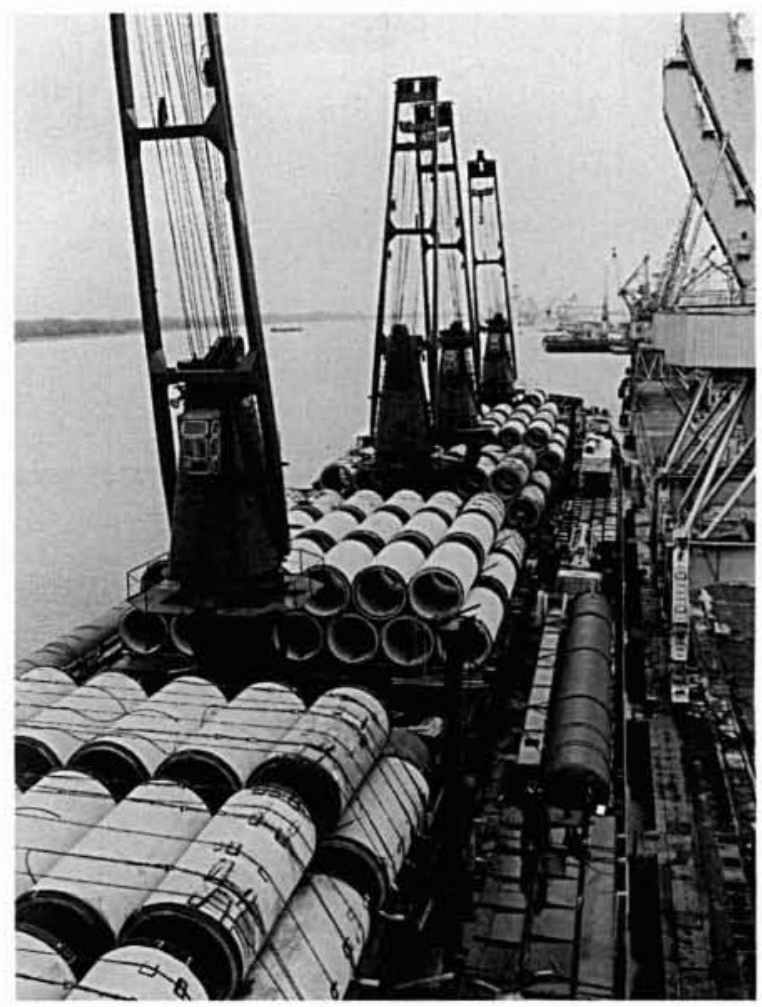

12. Tuyaux à âme en tôle pour l'exportation.

\section{VIII —LES FILIALES ÉTRANGÈRES DU GROUPE BONNA}

Face à la crise qui sévit dans les pays industrialisés, l'effondrement des prix, le recul de son marché intérieur et une concurrence internationale féroce, Bonna a choisi d'intensifier sa stratégie de diversification géographique en s'implantant de façon durable dans les pays en voie de développement pour être efficace là où il faut se battre, en développant des relations commerciales de proximité pour mieux s'adapter aux particularités locales, en étant mobile et en état de veille permanente. C'est ainsi qu'elle est présente en Belgique, en Hollande, en Espagne, en GrandeBretagne, en Suisse, en Tunisie, au Liban, en Egypte, en Afrique du Sud, au Mexique, en Indonésie et en Thaïlande, par le biais d'établissements ou de filiales. La filiale indonésienne, Humex Bonna Indonesia, créée en association avec le fabricant japonais Nippon Hume, remporte son premier contrat, en août 1993, qui porte sur la fabrication du circuit de refroidissement de la centrale de Black Point (Hong Kong). Attentive à l'essor du Sud-Est Asiatique, Bonna a intensifié sa présence en Asie et vient de créer une nouvelle filiale en Thaïlande. 